C-A/AP/\#184

December 2004

\title{
Transition Pressure Rise in RHIC Run-4
}

\author{
W. Fischer and U. Iriso
}

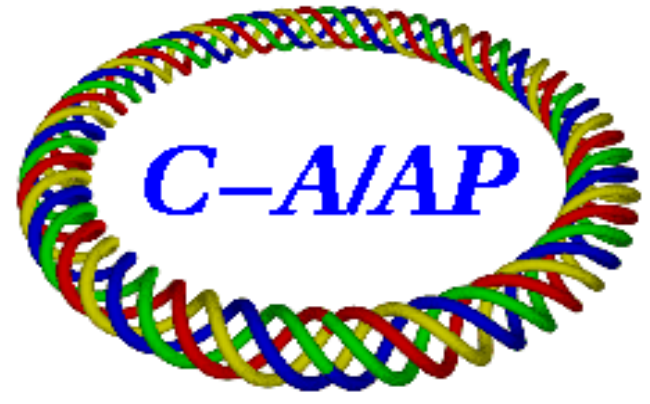

Collider-Accelerator Department Brookhaven National Laboratory Upton, NY 11973 


\title{
Transition Pressure Rise in RHIC Run-4
}

\author{
W. Fischer and U. Iriso
}

December 16, 2004

\begin{abstract}
During the RHIC Run-4 pressure rises could be observed in some of the interaction regions when the gold beams crossed the transition energy. We analyze these pressure rises as a function of location, store number, intensity, beam loss, and fill pattern. These findings are compared with electron cloud simulations. Our aim is to determine the dominant source of the transition pressure rise.
\end{abstract}

\section{Introduction}

Pressure rises in heavy ion operation are relevant at injection, transition, and store. They limit the intensity that can be injected, accelerated and stored, and lead to an increase in experimental background. While pressure rises at injection and store could clearly be linked to electron clouds [1-3], the source of the transition pressure is less clear. In Run3 the transition pressure rise was clearly dependent on the total intensity, but showed little or no dependence on the bunch pattern [4]. Beam loss was suspected as a possible source for the release of gas molecules. Measurements at BNL, CERN, and GSI have yielded desorption coefficients from 10 to $10^{7}$ for ions in the energy range from $1 \mathrm{MeV} / \mathrm{u}$ to $158 \mathrm{GeV} / \mathrm{u}$ (see Ref. [5] for a compilation of the data). The values at the high end of this range are still under discussion. Further desorption measurements were done at RHIC during Run-4 [6,7].

Here we analyze the transition pressure rise of all ramps that lead to physics stores in gold-gold operation in Run-4 (for an overview of all Runs, see [8]). For each case we record the following quantities:

1. Bunch pattern

2. Blue and Yellow intensity before transition

3. Blue and Yellow intensity after transition

4. Pressure in IR4, IR10, and IR12 before transition

5. Maximum pressure in IR4, IR10, and IR12 near transition

We analyze the pressure in the common sections of IR4, IR10, and IR12. In the common sections the pressure rise is usually more pronounced. In most cases only small or no pressure rises are seen during transition crossing in IR2, IR6, and IR8. In the following we discuss electron clouds and beam losses as possible sources for the transition pressure rise. 

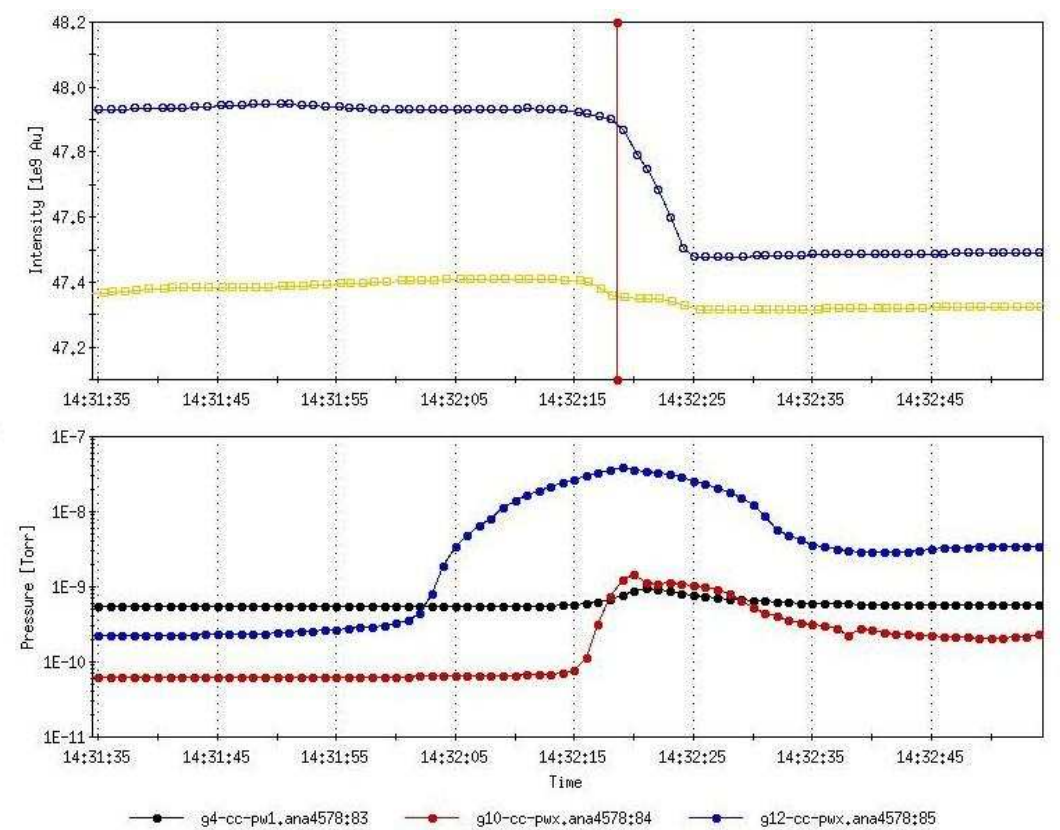

Figure 1: Typical intensities (top) and pressures (bottom) in IR4, IR10, and IR12 during transition crossing, indicated by the marker near 14:32:20.

\section{Observations}

We now discuss the transition pressure rise with respect to the location in the ring, the time structure, the beam intensity, the beam loss, and the bunch pattern. Fig. 1 shows a typical case of the transition pressure rise. When the energy approaches the transition energy, the pressure increases in IR12, later in IR10, and to a lesser extent in IR4. In IR12 the pressure increase happens before any beam loss is measurable.

Location Transition pressure rises are visible only in the warm regions. They can be observed in the common sections of IR12, IR10, and to a lesser extent in IR4. In the common parts of the other interaction regions as well as some other warm regions (notably yo1, yo4, and bi8), pressure rises are only observable for very high intensities.

Time structure The pressure rise in most cases precedes, and sometimes coincides with visible beam loss (see Fig. 1). The pressure usually drops back after transition crossing.

Time during Run-4 The transition pressure rise for all $\mathrm{Au}-\mathrm{Au}$ physics stores of Run-4 is shown in Fig. 2. Although there appear to be more problems at the beginning of the Run, there is no strong general trend. Stores 1 through 35 had 56 bunches per ring, stores 36 through 53 had 61 bunches/ring, stores 62 to 132 had 56 bunches/ring, and stores 133 through 274 had mostly 45 bunches/ring. We also note that stores 246 to 274 were at $31.2 \mathrm{GeV} / \mathrm{u}$ beam energy. The beams cross transition under the same conditions as for the $100 \mathrm{GeV} / \mathrm{u}$ beam energy. 
Intensity Fig. 3 shows the transition pressure rise as a function of the total beam intensity (Blue intensity plus Yellow intensity). There is a threshold of $85 \times 10^{9} \mathrm{Au}$ ions below which no transition pressure rise can be observed. Above this threshold there is an stronger than linear dependence of the pressure with the intensity. This is in agreement with the transition pressure rise observations in Run-3 [4].

Beam loss Fig. 4 shows the transition pressure rise as a function of the transition beam loss. There appears to be no correlation between the pressure rise and the amount of beam lost.

Bunch pattern Finally, Fig. 5 shows the maximum pressure near transition as a function of the average bunch intensity, separated into stores with 45, 56, and 61 bunches per ring, for IR10 and IR12. The threshold below which no pressure rise can be seen increases with the average bunch spacing. The pressure rise above the threshold is approximately linear.

\section{Electron induced desorption}

In this section we will argue that electron induced desorption, after an electron cloud formation, can explain the observed transition pressure rise. Figure 3 shows that the maximum pressure is strongly dependent on the total beam intensity, and Fig. 5 marks three different bunch intensity thresholds for this pressure rise corresponding to 45, 56, and 61 bunches per ring. For both IR10 and IR12 the threshold increases as the number of bunches per ring decreases (or the average bunch spacing increases). This is typical for electron clouds, not only for RHIC $[2,10]$ but also for other machines [9].

For electron clouds with a constant bunch pattern, we expect a density approximately proportional to the bunch intensity [10]. With an increase in the bunch intensity, the electron impact energy also increases (see below), and with it the desorption coefficient $\eta_{e}$ (number of molecules released per incident electron) [11]. We therefore expect a pressure rise dependence on the bunch intensity stronger than linear. This is consistent with the data shown in Fig. 5.

To gain further inside we simulate the electron cloud behavior in IR10 and IR12 for bunch patterns with 45, 56, and 61 bunches per ring. We consider the bunch lengths at injection, transition, and store. We used the code CSEC by M. Blaskiewicz (a description can be found in [8]). The simulation parameters are listed in Tab. 1. As observation point we chose a location close to a vacuum gauge, which is different in both IRs (Tab. 1), and we also assume for simplicity that the abort gaps coincide at both IRs. The pipe material in IR10 is beryllium, it is stainless steel in IR12. We choose for the total intensity $90 \times 10^{9}$ $\mathrm{Au}$ ions, a value at which we observe a clear pressure rise in both IR10 and IR12 for all bunch patterns (see Figs. 3 and 5).

The surface parameters related to the Secondary Electron Yield (SEY, $\delta$ ) are a critical ingredient to the simulation. Unfortunately and as seen in Ref. [14,15], there is a significant uncertainty in the SEY behavior as a function of the electron energy (specially at low energy) and the electron dose. Both dependences have a large influence in the studied 
Table 1: List of input parameters for electron cloud simulations at IR10 and IR12.

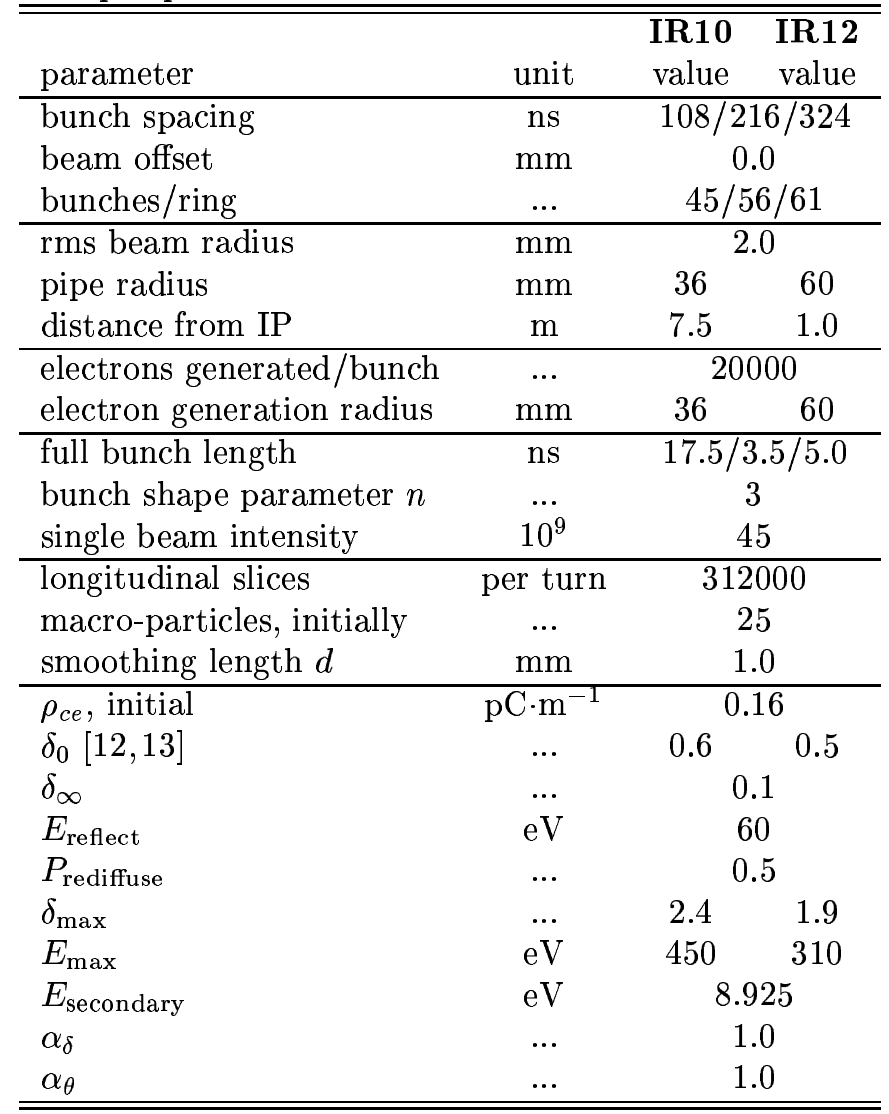

cases. As an example we mention that a $10 \%$ variation in the elastically reflection probability of low energy electrons (parameter $\delta_{0}$ in Tab. 1), or the maximum SEY (parameter $\delta_{\max }$ in Tab. 1), or even the energy at which $\delta_{\max }$ occurs (parameter $\mathbf{E}_{\max }$ in Tab. 1) can change the electron density by more than $100 \%$.

The surface parameters listed in Tab. 1 correspond to typical values found for stainless steel, OFHC and beryllium [12-16]. The final values were chosen to reproduce the observation that there is no electron cloud at injection with a full parabolic bunch length of $\sigma_{z}=17.5 \mathrm{~ns}$, and an electron cloud at transition $\left(\sigma_{z}=3.5 \mathrm{~ns}\right)$ and store $\left(\sigma_{z}=5 \mathrm{~ns}\right)$.

In Tab. 3 the simulation results are shown for IR10. We have effectively no electron cloud for the injection conditions. For transition and store conditions, when going from 61 to 56 to 45 bunches per ring, there are some variations in the electron cloud density, and electron flux into the wall. The energy of the cloud electrons increases monotonically when going to a smaller bunch number with larger bunch intensity. Note that the shorter the bunch length, the larger electron energy.

Tab. 3 shows the results for IR12. Here too we have no electron cloud for the injection conditions. For transition and store conditions, when going from 61 to 56 to 45 bunches per ring there is small a reduction in both the electron cloud density and the electron 
flux into the wall. The difference in the electron energy for different fill patterns is more pronounced than at IR10. This can be best seen in the maximum electron energy which rises, for the transition case, from about $500 \mathrm{eV}$ for 61 bunches per ring to about $700 \mathrm{eV}$ for 45 bunches per ring. At IR10 the maximum electron energy is about $100 \mathrm{eV}$ lower.

An increase in the electron energy could lead to a higher pressure rise if the desorption coefficient increases with the electron energy. In Fig. 6 (from Ref. [11]) molecular desorption coefficients are shown for electron energies up to $300 \mathrm{eV}$. In this range they are monotonically increasing. We have not found measurements at higher energies, and it is possible that the desorption coefficients do not or only slightly increase further with higher electron energies. The intensity scaling of the transition pressure rise is within expectations from electron clouds as the main source of the pressure rise.

In our simulations the electron cloud densities and currents into the wall change only slightly with the bunch pattern. We do see, however, a monotonic increase of the electron energy with a reduced bunch number and the same total intensity. This can compensate for a reduced electron flux into the wall and reproduce the total beam intensity behavior seen in Fig 3. Together with the change in the bunch intensity threshold observed in Fig 5 when moving from 61 to 56 to 45 bunches per ring, we therefore conclude that electron clouds can explain the observed transition pressure rises.

We note that it may be possible to calibrate the simulations such that they reproduce the observed bunch intensity thresholds as a function of the bunch pattern. With this, the simulations could yield useful information for setting intensity threshold during operation.

\section{Beam loss induced desorption}

In this section we will argue that beam loss induced desorption cannot explain the observed transition pressure rise. Fig. 4 shows that there is no correlation between the transition

pressure rise and visible beam losses. Nevertheless, beam losses in the form of halo scraping were proposed as the main cause of the transition pressure rise (see, for example, Ref. [17]). For beam losses to be responsible for the transition pressure rise one must assume that

1. The pressure rise is caused by halo losses not visible in the DCCT,

2. That these halo losses can occur in the IRs while the bulk losses occur at other locations in the ring, and

3. There is a sufficiently large desorption coefficient $\eta_{\text {loss }}$ for beam loss driven desorption.

The first point is necessitated by the observation shown in Figs. 1 and 4. The transition pressure rise can be seen without any visible beam loss. The second point implies that the beam halo losses are single-turn losses. Multi-turn losses, from a limited dynamic aperture, tend to be concentrated in a few locations of the ring (the physical aperture). Tab. 2 shows that the IRs between the outside of the DX magnets are not the limiting betatron aperture. Due to the small dispersion they are also not the limiting momentum 
Table 2: Comparison of betatron and momentum aperture in the RHIC arcs and interaction regions.

\begin{tabular}{lcccc}
\hline \hline & $\beta_{\mathrm{x}, \max }$ & $\beta_{\mathrm{y}, \max }$ & $\mathbf{D}_{\mathrm{x}, \max }$ & $d_{\text {pipe }}$ \\
{$[\mathrm{m}]$} & {$[\mathrm{m}]$} & {$[\mathrm{m}]$} & {$[\mathrm{cm}]$} \\
\hline arc & 48 & 48 & 1.5 & 7 \\
DX(outside)-DX(outside) & 45 & 45 & 0.03 & 12 \\
\hline \hline
\end{tabular}

aperture. The most likely single-turn loss mechanism is the electromagnetic interaction of a beam ion with the nucleus in one of the rest gas molecules. This loss mechanism is, however, not enhanced at transition. Other processes like electron capture or stripping, which dominate the instantaneous losses in lower energy ion accelerators, are not relevant in RHIC. We see therefore no loss mechanism that can account for halo scraping in the interaction regions, enhanced at transition.

For the third point we make a rough estimate for the required desorption coefficient $\eta_{\text {loss }}$ for beam loss driven desorption, assuming halo scraping as the main cause of the pressure rise. The pressure rise is approximately

$$
\Delta p=\frac{k T}{2 \pi r^{2}} \frac{d N}{d l} \eta_{\mathrm{loss}}
$$

where $k$ is the Boltzmann constant, $T$ the temperature, $r$ the beam pipe radius, and $d N / d l$ the particle loss per length. For a conservative estimate we assume that $10^{8} \mathrm{Au}$ ions (still visible in the DCCT) are lost within $10 \mathrm{~m}$ in IR12 leading to a pressure rise of $10^{-7}$ Torr. For this a desorption coefficient of $\eta_{\text {loss }} \approx 10^{7}$ is needed. This is at the high end of all observations, and was only inferred from some RHIC observations [5,6,18]. From the above we conclude that beam loss induced desorption cannot explain the observed transition pressure rise.

\section{Conclusions}

Significant transition pressure rises were observed in IR10 and IR12 during Run-4. These pressure rises can be explained assuming electron clouds as the driving mechanism. They cannot be explained assuming halo scraping during transition.

Since the transition pressure rises can be accounted for by electron clouds, we conclude that all operationally relevant pressure rises (at injection, transition, and store) can be explained by electron clouds. Beam loss driven desorption can lead to large pressure rises, as recent measurements in RHIC have confirmed [6,7]. However, in these instances the pressure rise is likely only the result of the large beam loss, which itself is an operational anomaly unrelated to the pressure rise.

\section{Acknowledgments}

The authors are tankful for discussions to M. Blaskiewicz, H. Huang, H.C. Hseuh, V. Ptitsyn, T. Roser, P. Thieberger, D. Trbojevic, J. Wei, and S.Y. Zhang. 


\section{References}

[1] U. Iriso, A.K. Drees, W. Fischer, D. Gassner, O. Gould, J. Gullotta, P. He, H.C. Hseuh, R. Lee, V. Ponnaiyan, L. Smart, D. Trbojevic, and S.Y. Zhang, "Electron Cloud Observation at RHIC in Run-3 ( 2002/03)", BNL C-A/AP/129 (2003).

[2] W. Fischer, M. Blaskiewicz, P. He, H. Huang, H.C. Hseuh, U. Iriso, G. Rumolo, L. Smart, D. Trbojevic, and S.Y. Zhang, "Electron Clouds and Vacuum Pressure Rise in RHIC", proccedings of the ECLOUD'04 Workshop, Napa, California, BNL-724512004-CP (2004).

[3] G. Rumolo and W. Fischer, "Observations on background in PHOBOS and related electron cloud simulations", BNL C-A/AP/146 (2004).

[4] S.Y. Zhang, "Experiment background in RHIC deuteron-gold Run", BNL C$\mathrm{A} / \mathrm{AP} / 107$ (2003).

[5] E. Mahner, I. Eftymiopoulos, J. Hansen, E. Page, H. Vincke, "Beam-loss induced pressure rise of LHC collimator materials irradiated with $158 \mathrm{GeV} / \mathrm{u} \mathrm{In}^{49+}$ ions at the CERN SPS", submitted to Phys. Rev. ST Accel. Beams (2004).

[6] U. Iriso, P. He, H.C. Hseuh, H. Huang, V. Ptitsyn, L. Smart, P. Thieberger, D. Trbojevic, "Interpretation of desorption measurements for high energy beams at RHIC", BNL C-A/AP/178 (2004).

[7] W. Fischer, H.C. Hseuh, U. Iriso, and E. Mustafin, "Gas desorption of stainless steel from irradiation with $9 \mathrm{GeV} / \mathrm{u} \mathrm{Au}^{79+}$ ions under perpendicular impact", in preparation (2004).

[8] W. Fischer, "Run overview of the Relativistic Heavy Ion Collider", http://www.rhichome.bnl.gov/RHIC/Runs/ (2004).

[9] F. Zimmermann, Presentation at "Panel discussion on future needs and future directions", ECLOUD'04 Workshop, Napa Valley, April 2004.

[10] W. Fischer, J.M. Brennan, M. Blaskiewicz, and T. Satogata, "Electron Cloud Measurements and Simulations for the Brookhaven Relativistic Heavy Ion Collider", Phys. Rev. ST Accel. Beams 5, 124401 (2002).

[11] F. Billard, N. Hilleret, G. Vorlaufer, "Some results on the electron induced desorption yield of OFHC copper", CERN LHC/VAC/FB/NH/GV00-32 (2000).

[12] B. Henrist, N. Hilleret, M. Jimenez, C. Scheuerlein, M. Taborelli, G. Vorlaufer, "Secondary Electron Data for the Simulation of Electron Cloud", proceedings of the ECLOUD'02 Workshop at CERN, CERN Yellow Report CERN-2002-001 (2002).

[13] M.A. Furman and M. Pivi, "Microscopic probabilistic model for the simulation of secondary electron emission", LBNL-49711, CBP Note-415 (2002). 
[14] R.E. Kirby, "Instrumental effects in secondary electron yield and energy distribution measurements", Proceedings of ECLOUD'04 at Napa Valley, 2004.

[15] R. Cimino, I. Collins, M. Furman, M. Pivi, G. Rumolo, F. Zimmermann, "Can low energy electrons affect high energy accelerators?", Phys. Rev. Lett. 93, 014801 (2004).

[16] N. Hilleret and B. Henrist, "SEY of Be" CERN LHC/VAC/TN-00-025 (2000).

[17] S.Y. Zhang, J. Alessi, M. Bai, M. Blaskiewicz, P. Cameron, A. Drees, W. Fischer, R. Fliller III, D. Gassner, J. Gulotta, P. He, H.C. Hseuh, H. Huang, U. Iriso, R. Lee, Y. Luo, W.W. MacKay, C. Montag, B. Oerter, S. Peggs, F. Pilat, V. Ptitsyn, T. Roser, T. Satogata, L. Smart, P. Thieberger, D. Trbojevic, L. Wang, J. Wei, J. van Zeijts, and K. Zeno, "RHIC Pressure Rise", proceedings of the 2004 European Particle Accelerator Conference, Lucerne, BNL-72062-2004-CP (2004).

[18] S.Y. Zhang, "Ion Desorption at RHIC", BNL C-A/AP/182, submitted to the proceedings of the 33rd ICFA Beam Dynamics Workshop on High Intensity and High Brightness Beams, Bensheim, Germany (2004). 

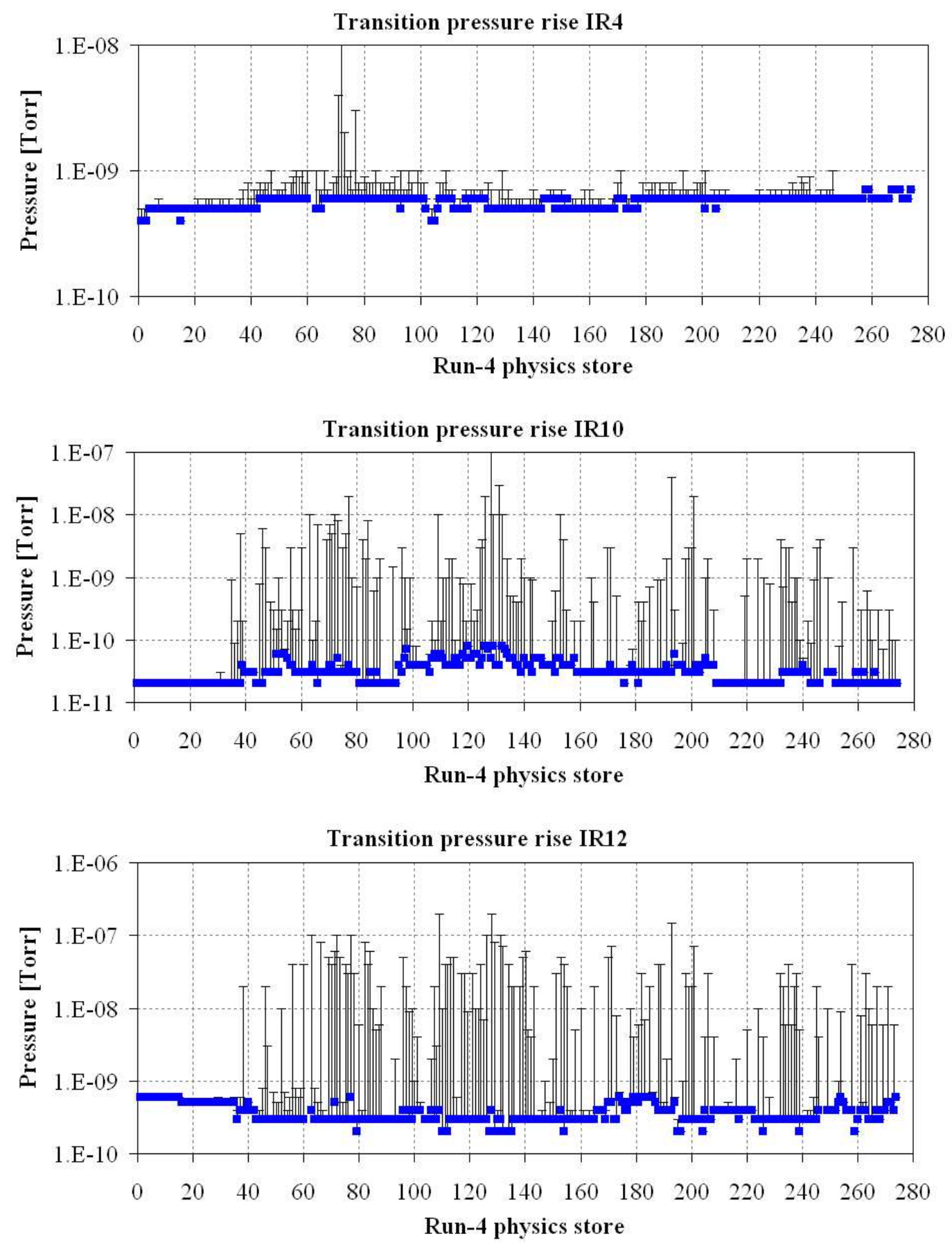

Figure 2: Transition pressure rise in IR4 (top), IR10 (middle), and IR12 (bottom) as a function of store number. Shown are all gold-gold physics stores of the RHIC Run-4. The blue squares show the pressure before transition is crossed, and the line the extend of the pressure rise at or shortly after transition. 

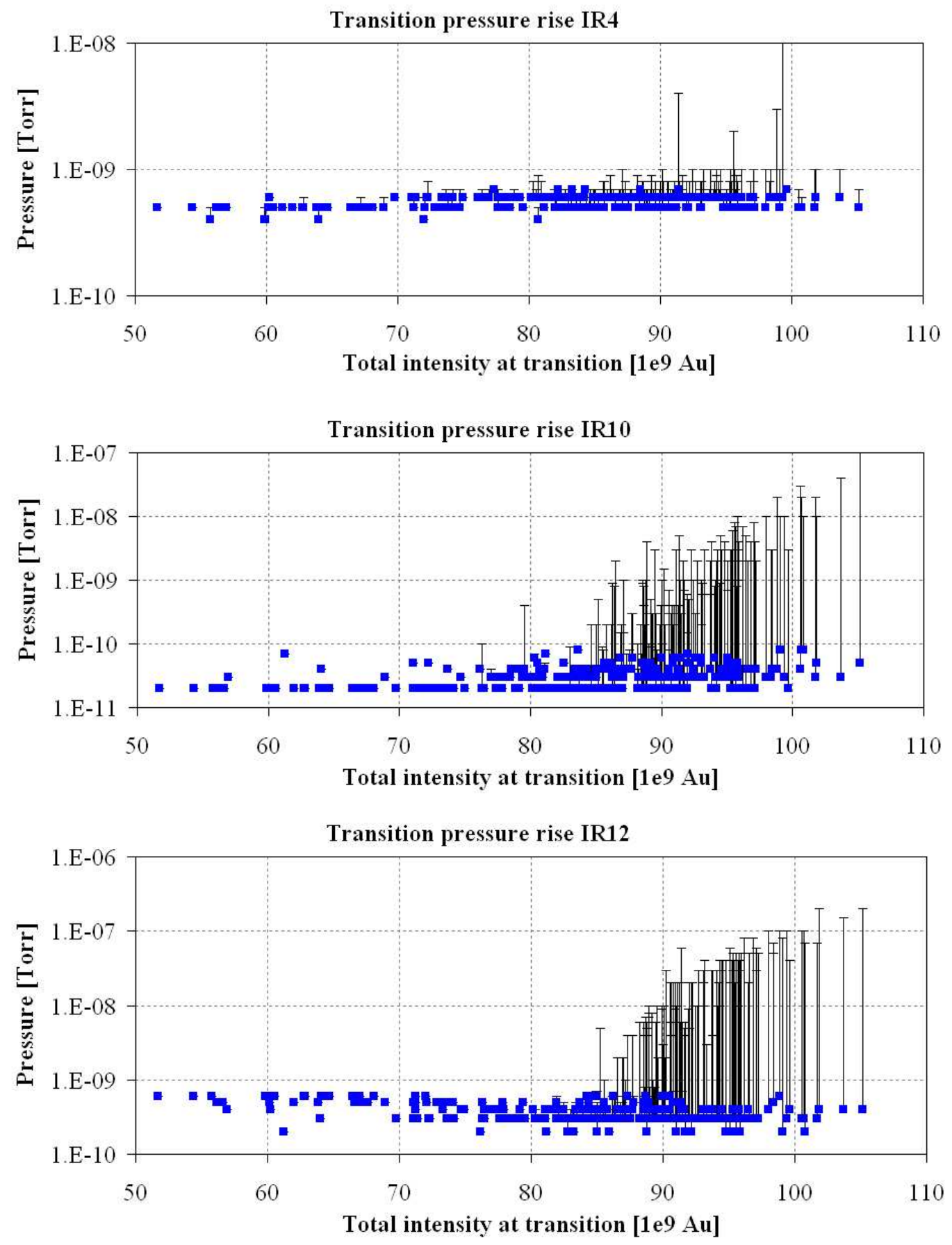

Figure 3: Transition pressure rise in IR4 (top), IR10 (middle), and IR12 (bottom) as a function of the total intensity. The total intensity is the sum of Blue and Yellow intensity, averaged over the values before and after transition. The blue squares show the pressure before transition is crossed, and the line the extend of the pressure rise at or shortly after transition. 

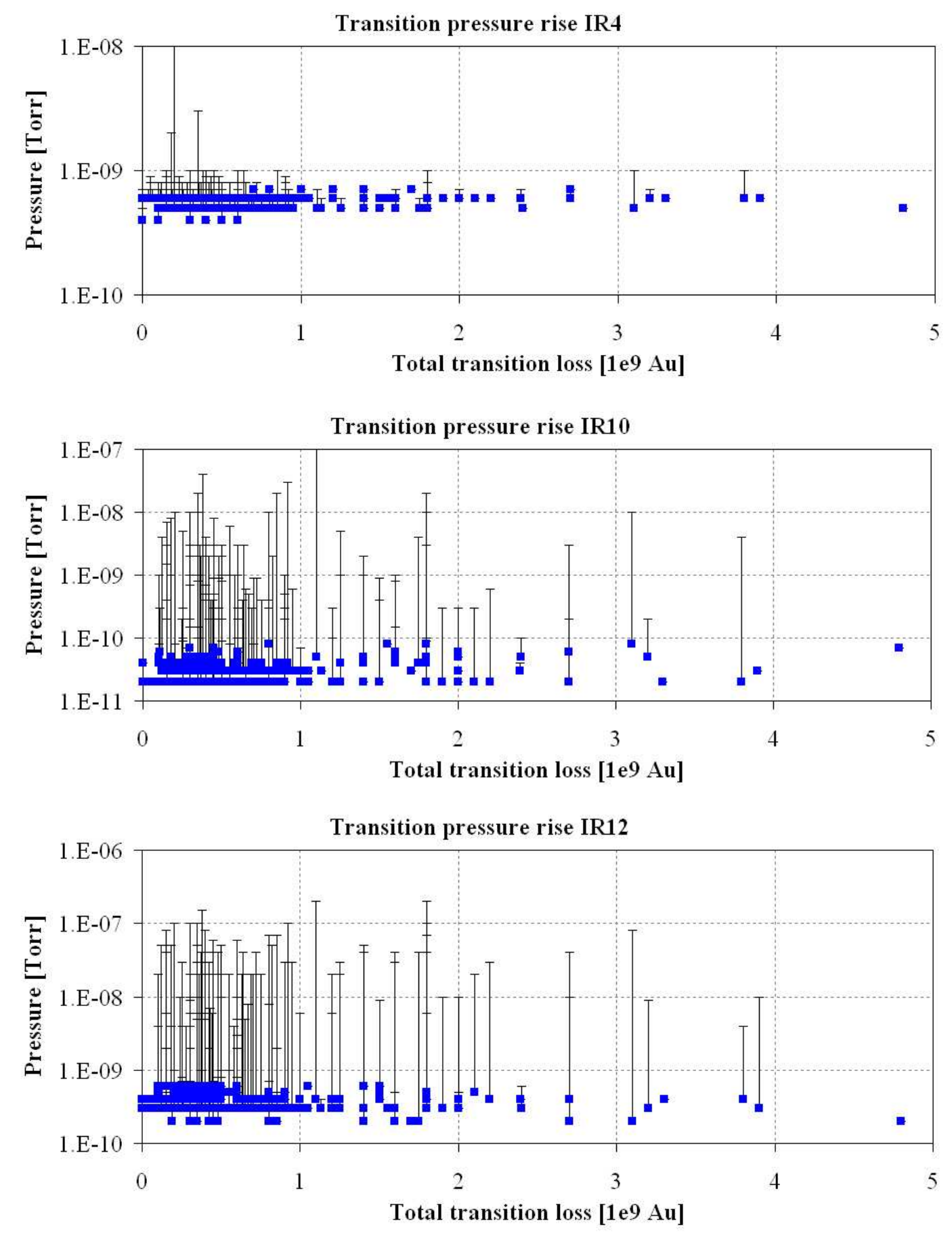

Figure 4: Transition pressure rise in IR4 (top), IR10 (middle), and IR12 (bottom) as a function of the total beam loss. The total beam loss is the sum of th Blue and Yellow beam loss near transition. The blue squares show the pressure before transition is crossed, and the line the extend of the pressure rise at or shortly after transition. 

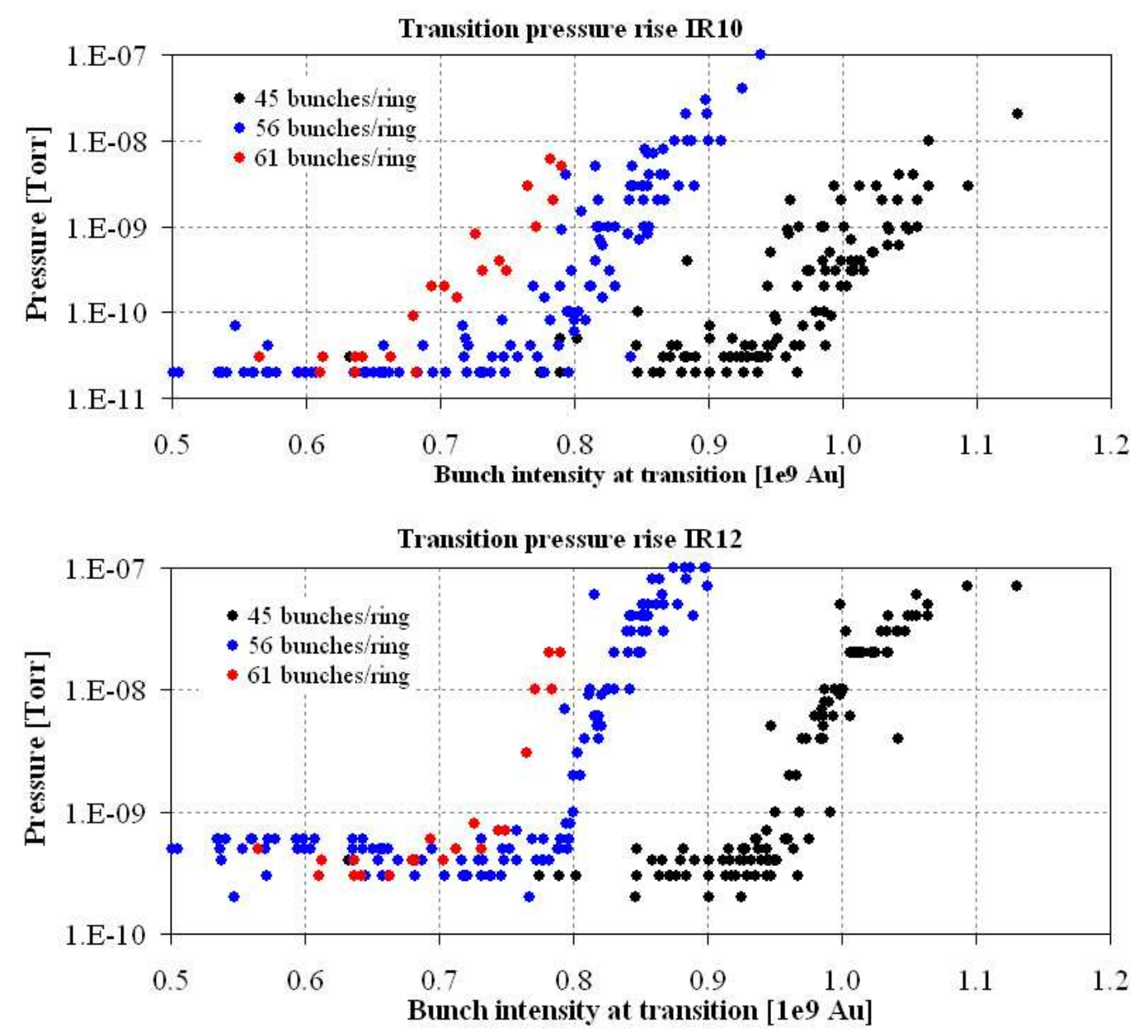

Figure 5: Transition pressure rise in IR10 (top), and IR12 (bottom) as a function of the average bunch intensity. The bunch intensity is averaged over the Blue and Yellow ring intensities, and the values before and after transition. The data is further separated into ramps with 45, 56, and 61 bunches per ring. The dots show the maximum pressure at or shortly after transition. 


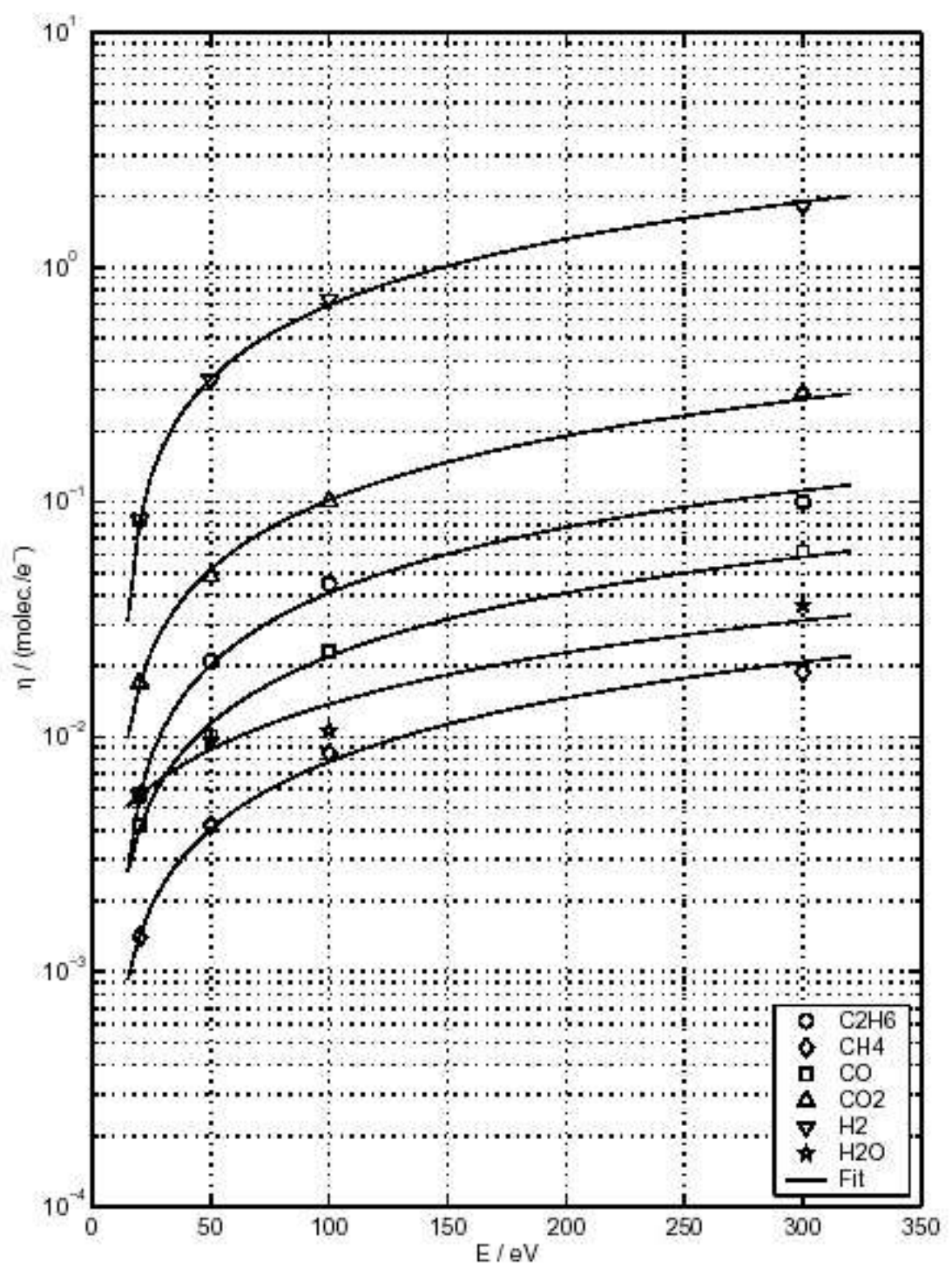

Figure 6: Electron desorption yield as a function of electron energy for OFHC copper (from Ref. [11]). 

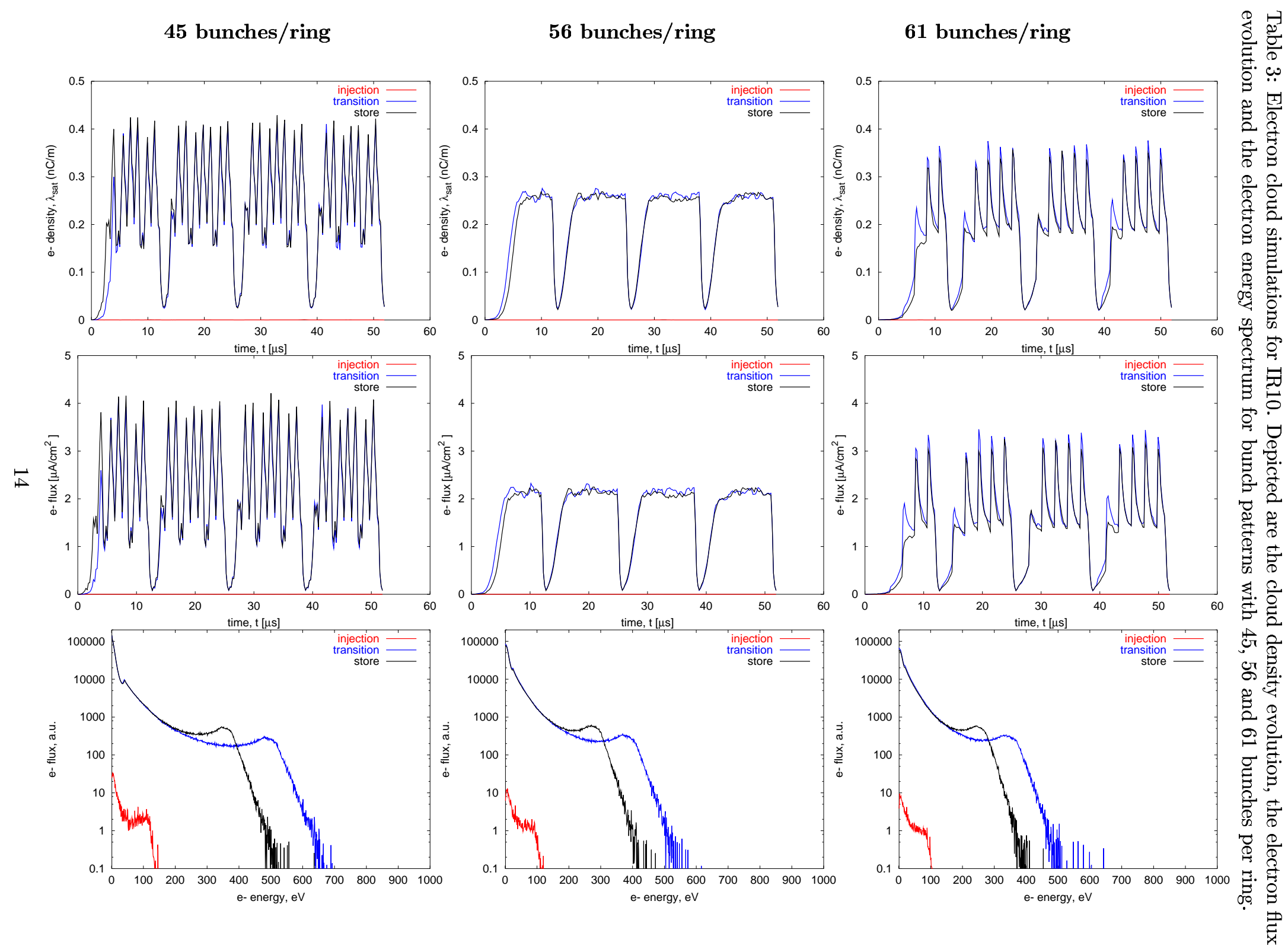

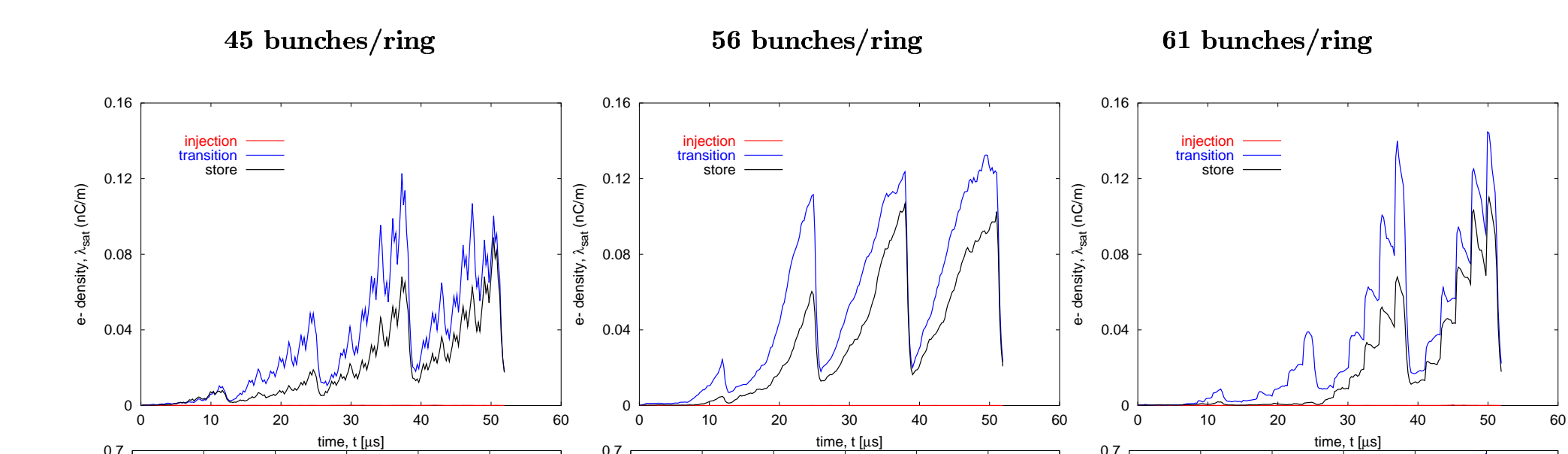

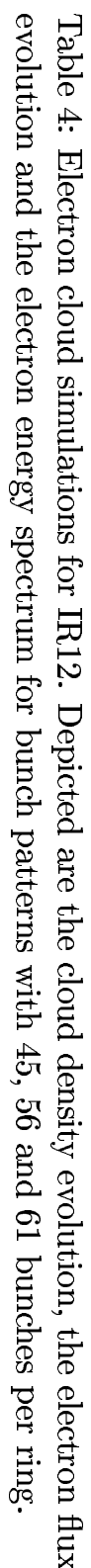
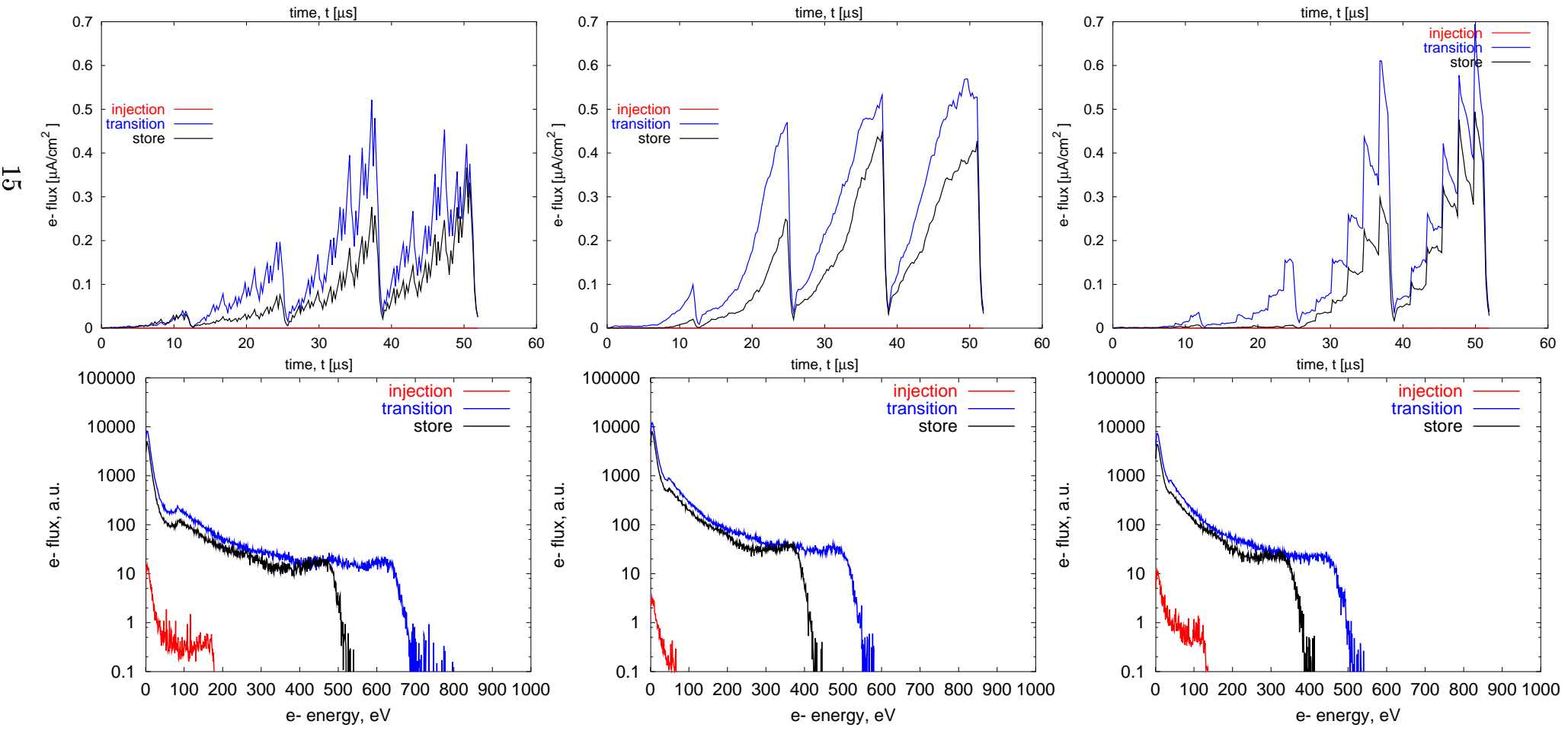
e- energy, eV 